This item was submitted to Loughborough's Research Repository by the author.

Items in Figshare are protected by copyright, with all rights reserved, unless otherwise indicated.

\title{
Measurement of sub-surface delaminations in carbon fibre composites using high-speed phase-shifted speckle interferometry and temporal phase unwrapping
}

PLEASE CITE THE PUBLISHED VERSION

PUBLISHER

(C) Elsevier

VERSION

AM (Accepted Manuscript)

LICENCE

CC BY-NC-ND 4.0

\section{REPOSITORY RECORD}

Alvarez, Abundio Davila, Pablo D. Ruiz, Guillermo H. Kaufmann, and Jonathan M. Huntley. 2019.

"Measurement of Sub-surface Delaminations in Carbon Fibre Composites Using High-speed Phase-shifted Speckle Interferometry and Temporal Phase Unwrapping". figshare. https://hdl.handle.net/2134/5931. 
This item was submitted to Loughborough's Institutional Repository (https://dspace.lboro.ac.uk/) by the author and is made available under the following Creative Commons Licence conditions.

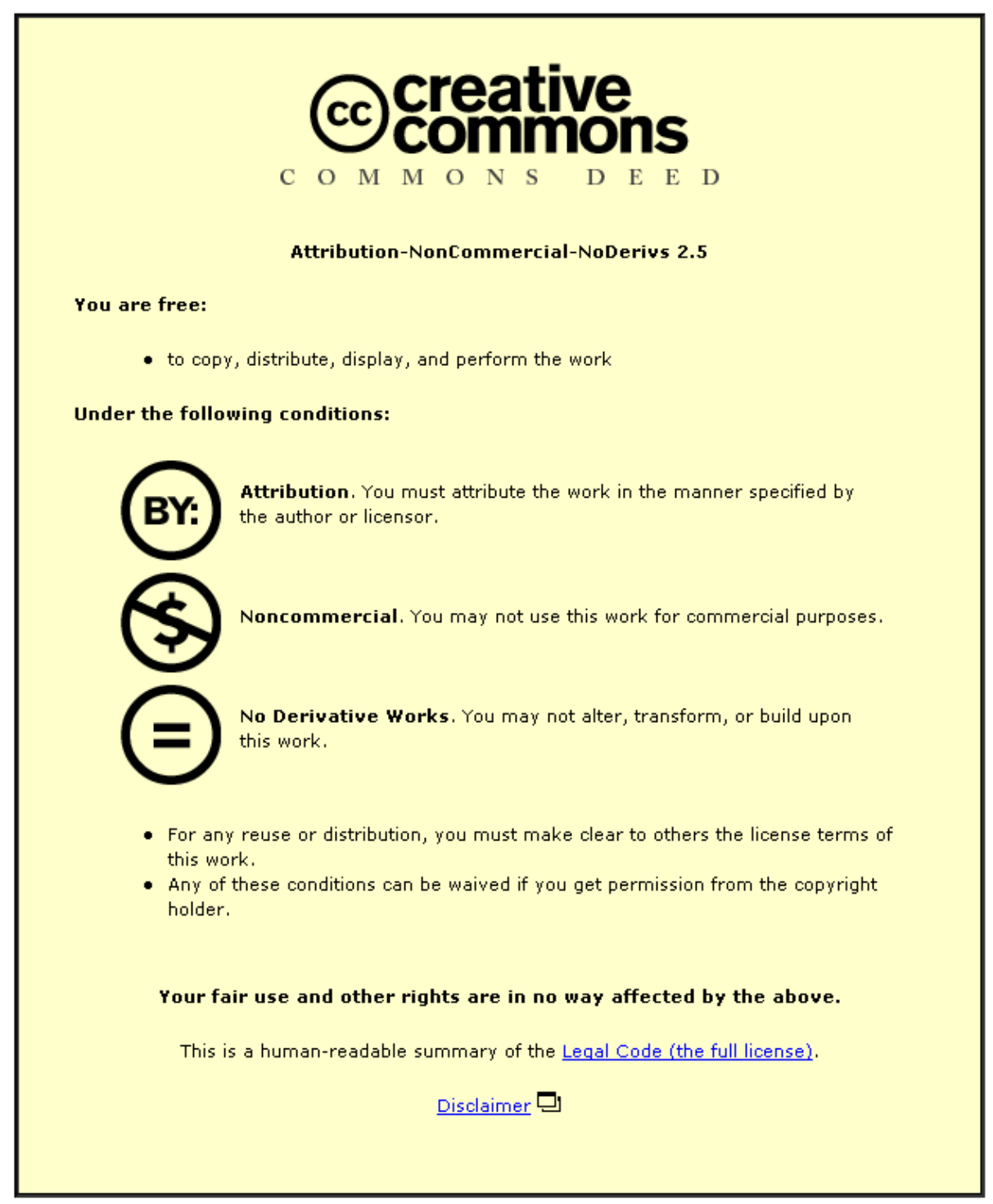

For the full text of this licence, please go to: http://creativecommons.org/licenses/by-nc-nd/2.5/ 


\title{
Measurement of sub-surface delaminations in carbon fibre
}

\section{composites using high-speed phase-shifted speckle interferometry and temporal phase unwrapping}

\author{
A. Davila[1], P. D. Ruiz[2], G. H. Kaufmann[2][3] and J. M. Huntley[1]
}

[1] Wolfson School of Mechanical and Manufacturing Engineering, Loughborough University, Loughborough, Leics. LE11 3TU, UK

[2] Instituto de Física Rosario (CONICET-UNR), 2000 Rosario, Argentina

[3] Departamento de Física, Facultad de Ciencias Exactas, Ingeniería y Agrimensura, Universidad Nacional de Rosario, 2000 Rosario, Argentina

\begin{abstract}
A high-speed phase-shifted speckle interferometer has been developed recently for studying dynamic events. Speckle interferograms are continuously recorded by a CCD camera operating at $1 \mathrm{kHz}$ with temporal phase shifting carried out by a Pockels cell running at the same frequency. Temporal phase unwrapping through sequences of more than 1000 frames allows the determination of time-varying absolute displacement maps. This paper presents the application of this speckle interferometry system to the detection and measurement of sub-surface delamination defects in carbon fibre specimens. The influence of re-referencing the temporal phase unwrapping algorithm after different time intervals is analyzed to reduce the random phase errors produced by speckle decorrelation and vibration. The performance of several phase shifting algorithms to minimize the influence of the vibration noise caused by the vacuum pump used to load the specimen is also investigated.
\end{abstract}


Keywords: Speckle Interferometry; Phase shifting; Phase measurement; Phase unwrapping; Optical metrology

\section{Introduction}

Electronic speckle pattern interferometry (ESPI) is a well-established technique for measuring displacement and strain fields of diffusely scattering objects, which was developed in 1971 [1]. However, it is since the introduction of phase shifting and computerized fringe analysis around the mid-1980s, that this whole-field optical technique has been developed into a powerful measurement tool for solving industrial and scientific problem [2]. It should be noted that this revival was also made possible by the increased computing power provided by new computers and by the recent advances of modern CCD detectors.

The data needed by end users of ESPI are obtained from measurements of the phase change distribution of speckle patterns recorded after and before deformation. Two main methods exist for extracting the underlying phase distribution: through Fourier transformation, and by introducing known phase shifts between the object and the reference waves [3]. Even though the analysis methods based on Fourier transformation of the whole image are quite popular, they have the significant drawbacks of reduced phase measurement accuracy and poorer spatial resolution compared to phase-shifting techniques. In both cases an arc-tangent function is used to calculate the speckle phase distributions, which are therefore wrapped onto the range $-\pi$ to $\pi$. The procedure used to restore the missing integral multiple of $2 \pi$ to the phase-change values, so as to remove the $2 \pi$ phase discontinuities, is known as phase unwrapping and is the most difficult step in the fringe analysis process [4].

The most common speckle interferometry analysis route used until recent time has involved phase shifting followed by spatial unwrapping. In this case, a known phase ramp, usually linear in position, is added to the phase function and all the data needed to calculate a 
phase map are acquired at a single time. It is worth pointing out that this method, which uses a single fringe pattern, is the preferred one in industrial applications because of its relatively high immunity to environmental disturbances. However, it has a reduced measurement range and problems arise when spatial phase unwrapping in two dimensions must be carried out. Noise and low modulated pixels can produce breaks in the phase steps and these can generate large phase errors when the unwrapping process is performed. When the object contains discontinuities, due for example to specimen edges or growing cracks, spatial unwrapping is also potentially risky because of the possibility of unwrapping across phase discontinuities which can cause the spatial propagation of a large phase errors.

One solution to these problems is to record a sequence of interferograms throughout the entire deformation history. In this case, the phase is calculated using temporal phase shifting by introducing a controlled phase change as a function of time. Using a sufficiently high temporal sampling rate, so that the intensity modulation along the time axis is sampled in accordance with Shannon's sampling theorem, each pixel can be unwrapped as a function of time [5]. As temporal phase unwrapping involves only one-dimensional signals, it is generally easier to carry out than spatial unwrapping. Also, temporal phase unwrapping allows absolute displacement fields to be obtained, which means the total displacement since the start of the measurement process. By contrast, spatial unwrapping can give only displacements relative to some other point in the field of view. Therefore, the combination of temporal phase shifting and temporal phase unwrapping means that each pixel (or small cluster of pixels, if spatial smoothing is carried out) in the camera behaves as an independent displacement sensor. Another advantage of this approach is that if a suitable re-referencing is chosen, the temporal phase unwrapping algorithm allows the measurement of large-object deformation up to a few hundred or more micrometers. 
A high-speed speckle interferometry system based on this concept was recently developed by Huntley et al [6]. This system uses a digital video camera running at $1 \mathrm{kHz}$, i.e. a factor of 40 times faster than can be achieved with conventional video cameras. Temporal phase shifting was carried out using a Pockels cell in the reference beam and phase extraction was carried out by the use of a standard 4-frame algorithm. Temporal unwrapping through sequences of more than 1000 frames was used to obtain time-varying absolute displacement maps.

The performance of this high-speed speckle interferometry system in the presence of harmonic vibrations was recently analyzed using controlled experiments and numerical simulations [7]. It was demonstrated that the frequency response of the rms phase error depends mainly on the form and length of the temporal window of the phase shifting algorithm and also on the framing rate of the video camera. The rms phase error that appears when the system is used with different phase shifting algorithms in the presence of random vibrations was analyzed in Ref. 8. It is shown that the unwrapping success rate given by different phase-shifting formulae depends mainly on the temporal duration of the algorithm window and also on the rms displacement amplitude of the vibration. Short window algorithms provide greater unwrapping reliabilities provided that the Nyquist velocity limit of the object is not exceeded.

The purpose of this paper is to describe the application of this novel speckle interferometry system to the detection and measurement of sub-surface delamination defects in carbon fibre composites specimens, similar to those used in the aerospace manufacturing industry. Also, this paper warns about two sources of phase error, i.e. vibration and speckle decorrelation, that particularly affect long recording sequences and gives advice on how to reduce their effects during phase evaluation and phase unwrapping. The deformation of the specimen was introduced by placing it in a vacuum chamber with a transparent window. To 
simulate the recording of interferograms in a noisy environment similar to the one existing outside of the laboratory, data were also acquired while the vibration-isolating-mechanism of the table over which the interferometer was mounted was left depressurised and the vacuum pump was running. In order to reduce the errors produced by random noise and speckle decorrelation, the influence of re-referencing the temporal phase unwrapping algorithm after different time intervals was analyzed. The performance of several phase shifting algorithms to minimise the influence of the vibration noise caused by the vacuum pump was also investigated.

\section{Experimental setup}

Figure 1 shows a schematic view of the high-speed phase-shifted speckle interferometer used to measure the out-of-plane displacement field generated by the carbon fibre specimen submitted to a vacuum load. The beam from a frequency-doubled continuouswave Nd:YAG laser (5W Verdi from Coherent, $\lambda=532 \mathrm{~nm}$, used at approximately $20 \%$ of its maximum power) is divided into the object and reference arms by a 90:10 beam splitter. The reference beam is passed through a Pockels cell and recombined with the speckle pattern formed by the scattered object beam using a second 90:10 beam splitter in front of the camera objective. The Pockels cell used here was manufactured by Leysop Ltd. as a phase modulator for this specific application. The Pockels cell is driven by a staircase waveform generator which produces one output of a set of four equally-spaced voltage levels, which are clocked cyclically in response to the rising edges of a TTL square wave input signal. A function generator is used to produce the square wave, which also drives the high-speed video camera, and therefore acts as the master clock for the whole experiment. The Pockels cell was calibrated to achieve phase shifts of $\pi / 2$ between successive frames by means of the procedure presented in Ref. 6 . 
The camera is a Kodak Ektapro model 1012 high-speed device, capable of acquisition rates of up to 1000 full frames per second. The frame store within the camera control unit is able to hold up to 1600 images at a resolution of $239 \times 192$ pixels $\times 8$-bit. The images are downloaded through a GPIB interface to a Sun SPARCstation for analysis.

The specimen consisted of a plate of $15 \times 10 \times 0.15 \mathrm{~cm}^{3}$, manufactured with a stacking sequence of $[0 / 90 / 0 / 0 / 90 / 0]$, which refers to the fibres angle between layers. A double-layer of Teflon film with dimension of $5 \times 5 \mathrm{~cm}^{2}$ was included between the first and second plies to simulate the presence of a delamination with intracavity air. After curing, the specimen was coated with titanium dioxide developer powder to increase the surface reflectivity. The plate was placed in a vacuum chamber with optical access provided by a polymethyl methacrylate window. The surface displacement field was measured with the high-speed speckle interferometer at a framing rate of 1000 frames $\mathrm{s}^{-1}$ as the pressure was reduced at an approximate rate of $2 \times 10^{4} \mathrm{~Pa} / \mathrm{s}$.

\section{Data analysis}

The measured intensity $I(t)$ at a given camera pixel follows the usual phase-shifting interferometry equation

$$
I(t)=I_{r}+I_{o}+2 \sqrt{I_{r} I_{o}} \cos [\Phi(t)+\phi(t)]
$$

where $I_{\mathrm{r}}$ and $I_{\mathrm{o}}$ are the reference and object beam intensities, $t$ is the frame number $(t=0,1,2$, $\left.\ldots, N_{t}-1\right)$, and $\Phi(t)$ is the speckle phase. $\phi$ is the phase shift introduced by the Pockels cell in the reference beam, which follows the repetitive cycle $(0, \pi / 2, \pi, 3 \pi / 2)$.

As stated in Ref. 5 , the phase change $\Delta \Phi_{\mathrm{w}}\left(t_{2}, t_{1}\right)$ between times $t_{1}$ and $t_{2}$, which is wrapped onto the range $(-\pi, \pi)$, is calculated from the equation 


$$
\Delta \Phi_{w}\left(t_{2}, t_{1}\right)=\tan ^{-1}\left\lceil\frac{N\left(t_{2}\right) D\left(t_{1}\right)-D\left(t_{2}\right) N\left(t_{1}\right)}{D\left(t_{2}\right) D\left(t_{1}\right)+N\left(t_{2}\right) N\left(t_{1}\right)}\right\rfloor,
$$

where $N(t)$ and $D(t)$ are respectively proportional to $\sin \Phi(t)$ and $\cos \Phi(t)$, and are given by

$$
N(t)=\operatorname{Im}[z(t)], \quad D(t)=\operatorname{Re}[z(t)]
$$

The complex variable $z(t)$ is defined by

$$
z(t)=\left[\sum_{t^{\prime}=0}^{M-1} a\left(t^{\prime}\right) I\left(t+t^{\prime}\right)+i \sum_{t^{\prime}=0}^{M-1} b\left(t^{\prime}\right) I\left(t+t^{\prime}\right)\right] \exp \left(-i \frac{\pi t}{2}\right)
$$

where the coefficients $a(t)$ and $b(t)$ depend on the particular phase shifting algorithm used to calculate $\Delta \Phi_{\mathrm{w}}(t)$, and $M$ is the number of frames used in the algorithm. The complex exponential term in Eq. (4) demodulates the temporal carrier signal in order to compensate for the initial phase offset in the first frame of any group of $M$ frames.

By defining

$$
a(t)+i b(t)=w(t) e^{-i \frac{\pi t}{2}}, \quad t=0,1,2, \ldots, M-1,
$$

it can be seen that the term in the square brackets in Eq. (4) is equivalent to the Fourier transform of a windowed intensity signal evaluated at the modulation frequency $k_{t}=1$ [9]

$$
\widetilde{I}\left(k_{t}, t\right)=\sum_{t^{\prime}=0}^{M-1} I\left(t+t^{\prime}\right) w\left(t^{\prime}\right) \exp \left(-2 \pi i k_{t} t^{\prime} / N\right)
$$

The window coefficients $w(t)$ can be easily calculated from the specified sampling coefficients $a(t)$ and $b(t)$, or vice versa, using Eq. (5). For example, in the case of a 4-frame phase shifting algorithm based on a rectangular window, ${ }^{6} M=4 ; w=[1,1,1,1], a=[1,0,-1$, $0]$ and $b=[0,-1,0,1]$.

It should be noted that Eq.(2) allows the calculation of the total wrapped phase change $\Delta \Phi_{\mathrm{w}}(t, 0)$ since the start of the recording process. A significant advantage offered by Eq. (2) over direct subtraction of separately-calculated phases is that its numerator and denominator are respectively proportional to the sine and cosine of the phase change between times $t_{1}$ and 
$t_{2}$, weighted by the product of the intensity modulations at the two times. Spatial smoothing can therefore be carried out by convolving the numerator and denominator with a $3 \times 3$ kernel of equal constant values. It can be shown that this smoothing results in a phase-difference map with optimal signal-to-noise ratio [10].

The unknown integral multiple of $2 \pi$ for each value of $t$ is found by temporal phase unwrapping. To determine the unwrapped phase change distribution $\Delta \Phi_{\mathrm{u}}(t, 0)$, the number of $2 \pi$ phase jumps between two successive measurements of the phase change is calculated by means of

$$
d(t)=\operatorname{NINT}\left\{\left[\Delta \Phi_{w}(t, 0)-\Delta \Phi_{w}(t-1,0)\right] / 2 \pi\right\}, \quad t=2,3, \ldots N_{t}-1,
$$

where NINT $\{x\}$ rounds $x$ to the nearest integer.

Then, the total number of phase jumps, $v(t)$, is calculated as

$$
\begin{aligned}
& v(t)=\sum_{t^{\prime}=2}^{t} d\left(t^{\prime}\right), \quad t=2,3, \ldots N_{t}-1, \\
& v(1)=0
\end{aligned}
$$

and finally the unwrapped phase difference map is obtained as

$$
\Delta \Phi_{u}(t, 0)=\Delta \Phi_{w}(t, 0)-2 \pi v(t), \quad t=1,2, \ldots N_{t}-1 .
$$

It should be pointed out that if the deformation or tilt becomes too large, significant speckle decorrelation will occur causing an increase in the noise level of the measured displacement fields. Under such circumstances, a new reference state should be chosen at times $t=t_{1}, t_{2}, t_{3}, \ldots$ in place of the state $t=0$. The total unwrapped phase change after rereferencing $\kappa$ times will then be

$$
\Delta \Phi_{u}(t, 0)=\Delta \Phi_{u}\left(t, t_{\kappa}\right)+\sum_{k=2}^{\kappa} \Delta \Phi_{u}\left(t_{k}, t_{k-1}\right)+\Delta \Phi_{u}\left(t_{1}, 0\right)
$$

It was shown in Ref. 6 that if re-referencing is carried out too frequently, bias and high noise levels will be introduced into the phase maps. On the other hand, a low rate of rereferencing could produce large random errors due to large speckle decorrelation. The effect 
of re-referencing and selection of its optimal rate were not, however, addressed in that publication and therefore form the main areas of investigation in the current paper. The rereferencing rate can of course be varied at the post-processing stage, allowing a systematic comparison of its effect on data quality to be made. For the results presented here, we kept the re-referencing rate constant throughout each post-processing run of a given dataset. The variable $\tau_{\mathrm{r}}=t_{\mathrm{k}}-t_{\mathrm{k}-1}$ is used to specify the number of frames between successive re-referencing events.

\section{Experimental results}

Figure 2 shows the wrapped phase difference map obtained for the carbon fibre specimen when the vibration-isolating table was pressurized and the vacuum pump was switched off. This wrapped phase difference map, in which black pixels represent $-\pi$ and white ones represent $+\pi$, was obtained from a set of 500 frames using Eq. (2) and the 4 -frame phase shifting algorithm for $t=500$. From this figure it is seen that the pattern due to the outof-plane displacement of the carbon fibre specimen is formed by a set of vertical fringes superimposed on a closed fringe pattern. The first pattern is caused by the specimen tilt while the second one is due to the expansion of the delamination generated by the vacuum loading. The location of the defect is clearly depicted in this image. It is also observed that because of large-object deformation, tilt and residual speckle noise, some areas of the phase map do not give reliable data [10]. The unwrapped phase distribution obtained by the temporal unwrapping without re-referencing $\left(\tau_{\mathrm{r}}=\infty\right)$ is shown in gray levels and also as a 3-D plot in Figs. 2(b) and 2(c), respectively. If the carbon fibre specimen is illuminated quasi normally, the unwrapped phase map can be converted to the out-of-plane displacement component $u_{z}(x, y)$ by multiplying by the constant $\lambda / 4 \pi=0.0423 \mu \mathrm{m} \mathrm{rad}^{-1}$.

The regions of highest slope in Fig. 2(a) have a spatial phase gradient of approximately 0.3 fringes per pixel. The $3 \times 3$ spatial convolution filter therefore averages over almost one complete fringe cycle, thereby reducing the modulation to a value close to 
zero in these regions of the image. As discussed later, this problem can be avoided by more frequent re-referencing of the speckle pattern. We include this figure, however, firstly to illustrate how valid data can be obtained even when operating close to this theoretical maximum phase gradient (of one fringe per three pixels), and secondly to demonstrate the benefits of temporal over spatial unwrapping. Even sophisticated 2-D spatial unwrapping algorithms would fail to provide an acceptable unwrapped phase map from Fig. 2(a), whereas Figs 2(b) and (c) show how the temporal unwrapping errors are constrained to the regions of the sample where the phase data is invalid.

The curve (a) of Fig. 3 shows the phase profile of the specimen displacement obtained along a horizontal line crossing the point of maximum deformation when the vibrationisolating-mechanism of the table was pressurized and the vacuum pump was disconnected. This result was obtained using again the 4-frame phase shifting algorithm after 1000 frames and re-referencing the temporal phase unwrapping algorithm every 5 frames $\left(\tau_{\mathrm{r}}=5\right)$. For comparison, the curve (b) of Fig. 3 shows the phase profile obtained by recording the interferogram sequence on the optical table with no vibration isolation while the vacuum pump was running (a phase offset was added to the figure data to better compare both curves). It is seen that the vacuum pump introduced a quite a large amount of vibration noise which contaminated the phase data.

The phase profiles through the unwrapped phase map obtained using the 4-frame phase shifting algorithm and 5 frames between re-referencing events $\left(\tau_{\mathrm{r}}=5\right)$ for $t=250,500$, 750 and 1000 are shown in Fig. 4. These results were obtained by recording the data with no vibration isolation while the vacuum pump was running. From this figure another advantage of temporal unwrapping (in addition to the lack of spatial error propagation) is clear: absolute displacements are obtained rather than the relative ones that would be determined by use of 
spatial. A further benefit of temporal unwrapping is that one obtains the time evolution of the displacement field, allowing one to improve the discrimination of different defects.

As was previously pointed out, if the deformation becomes too large, significant speckle decorrelation will occur between the two different sets of phase shifted interferograms. In this case, speckle decorrelation is mainly caused by out-of-plane tilts and also by strains (derivatives of displacements) of the specimen which generate speckle translation across the objective lens used to form the image. ${ }^{10}$ To illustrate the effect of rereferencing every $\tau_{\mathrm{r}}$ frames in the temporal phase unwrapping algorithm, Fig. 5 shows the phase profiles obtained for $t=1000$ with the 4-frame phase shifting algorithm for $\tau_{\mathrm{r}}=0,30,60,120,240$ and $\infty$ (no re-referencing) when the vibrations generated by the vacuum pump were present. These plots, which include artificial phase offset values for clarity, show that the random phase errors due to low fringe modulation in regions of high phase gradient decreases with the number of frames $\tau_{\mathrm{r}}$ used to re-reference the temporal phase unwrapping algorithm. From these results, it is also clear that an optimal number of frames $\tau_{\mathrm{r}}$ must exist. For the case of the 4-frame phase shifting algorithm, the results shown in Fig. 5 show that the optimal number of frames between re-referencing events lies in the interval from $\tau_{\mathrm{r}}=60$ to 120 frames.

To illustrate the performance of different phase shifting methods to minimise the random phase errors introduced by the vibration noise generated by the vacuum pump, Fig. 6 shows the phase profiles obtained using the following algorithms: 4-frame, 6-frame, Carré, rectangular window with $M=8$ (8-rect) and $M=16$ (16-rect) and Hanning window with $M=16$ (16-hann). ${ }^{7}$ These phase profiles are shifted by arbitrary offset values for clarity and were obtained for $\tau_{\mathrm{r}}=60$ with no vibration isolation and with the vacuum pump running. The experimental results suggest that short $M$-value phase-shifting algorithms, such as the Carré and the 8-rect ones, minimise the random phase errors introduced by the vibration noise. 
These preliminary conclusions agree with the conclusions presented in Ref. 8, where through a numerical simulation it is shown that short time window algorithms in the presence of random vibrations give grater phase unwrapping reliability, as long as the Nyquist velocity limit of the object is not exceeded. The phase unwrapping reliability is related to the ability to track the phase during the time history of the signal. In the same paper it is also shown that short window phase shifting algorithms give minimum average rms phase errors in order to obtain smooth unwrapped phase maps.

Finally, it should be pointed out that a study of the influence of different speckle decorrelation sources on the temporal unwrapping algorithm and the determination of the optimal number of frames $\tau_{\mathrm{r}}$ to be used to re-reference it, as function of the phase-shifting algorithm used to evaluate the wrapped phase changes, is beyond the scope of the present paper. The accumulation along the time axis of the small phase errors introduced in the phase evaluation by intensity fluctuations, velocity, and speckle decorrelation was recently analysed by means of computer generated speckle patterns. This study shows that the accumulation of phase errors yields total phase values that depend strongly on the re-referencing rate and also on the phase stepping algorithm. These results, and their comparison with those obtained from a controlled laboratory experiment, will be the subject of a forthcoming paper.

\section{Conclusions}

We have presented the application of a recently developed high-speed phase-shifted speckle interferometer to the detection of sub-surface delamination defects in carbon fibre specimens. The speckle interferometer allows the recording of a sequence of interferograms by means of a CCD camera operating at $1 \mathrm{kHz}$. Data are processed using a combination of temporal phase shifting and temporal phase unwrapping, allowing the determination of timevarying absolute displacement maps in excess of $100 \mu \mathrm{m}$. With this technique, each cluster 
formed by $3 \times 3$ pixels over the specimen surface behaves as an independent displacement sensor. The results obtained from a vacuum-loading test suggest interesting applications for non-destructive analysis. It is seen that re-referencing of the temporal phase unwrapping algorithm after different time intervals has a remarkable effect on the unwrapped phase, the main benefit being the extension of the measurement range without the limit normally imposed by speckle decorrelation. Even though a theoretical basis of the optimum rereferencing rate is still a research subject, for the tests performed in the vacuum chamber best results were obtained when re-referencing every 60 to 120 frames $\left(\tau_{r}=60-120\right)$, even in the presence of vibration and large displacements. Also, short time window algorithms provided better performance when evaluating the wrapped phase, showing low sensitivity to vibration. It is expected that the measurement of the time-varying displacement field generated by a delamination will allow in the near future the evaluation of its size and location in depth through the use of inverse analysis.

\section{Acknowledgements}

A. Davila is on leave from Centro de Investigaciones en Optica, Leon, Mexico. Support in the form of a research fellowship, equipment and staff costs was provided by Engineering and Physical Sciences Research Council under contracts GR/N22380 and GR/M57835. The authors are grateful to Fundacion Antorchas of Argentina and The British Council for the research award obtained to support the visits of P. D. Ruiz and G. H. Kaufmann to Loughborough. We also thank D. Panni for the carbon fibre sample and Y. Shen for assistance with the numerical analysis. 


\section{References}

[1] J. N. Butters and J. A. Leendertz, "Speckle pattern and holographic techniques in engineering metrology," Opt. Laser Technol. 3, 26-30 (1971).

[2] R. S. Sirohi (Ed.), Speckle Metrology, Academic Press, New York, 1993.

[3] J. M. Huntley, "Automated analysis of speckle interferograms," in Digital Speckle Pattern Interferometry and Related Techniques, P. K. Rastogi (Ed.), Wiley, Chichester, UK (2000), pp. 59-139.

[4] D. C. Ghiglia and M. D. Pritt, Two-Dimensional Phase Unwrapping, Wiley, New York, 1998.

[5] J.M. Huntley and H. Saldner, "Temporal phase-unwrapping algorithm for automated interferogram analysis," Appl. Opt. 32, 3047-3052 (1993).

[6] J. M. Huntley, G. H. Kaufmann and D. Kerr, "Phase-shifted dynamic speckle pattern interferometry at 1 kHz," Appl. Opt. 38, 6556-6563 (1999).

[7] P. D. Ruiz, J. M. Huntley, Y. Shen, C. R. Coggrave and G. H. Kaufmann, "Vibrationinduced phase errors in high-speed phase-shifting speckle pattern interferometry," Appl. Opt. 40, 2117-2125 (2001).

[8] P. D. Ruiz, J. M. Huntley, Y. Shen, C. R. Coggrave and G. H. Kaufmann, "Effects of random vibration in high-speed phase-shifting speckle pattern interferometry," Appl. Opt. (under review).

[9] P. de Groot, "Derivation of algorithms for phase-shifting interferometry using the concept of a data-sampling window," Appl. Opt. 34, 4723-4730 (1995).

[10] J. M. Huntley, "Random phase measurement errors in digital speckle pattern interferometry," Opt. Lasers Eng. 26, 131-150 (1997). 


\section{Figure Captions}

Figure 1. Optical set up of the high-speed out-of-plane speckle interferometer: frame store (F), Pockels cell (P), high voltage driver (D), function generator (G), 90/10 beam splitters (BS), mirrors (M) and lenses (L), carbon fibre specimen (S), vacuum chamber (V).

Figure 2. Results obtained for the carbon fibre specimen using the 4-frame phase shifting algorithm for $t=500$ with no re-referencing $\left(\tau_{\mathrm{r}}=\infty\right)$ in the temporal unwrapping algorithm, with vibration isolation and with the vacuum pump switched off: (a) Wrapped phase map; (b) Unwrapped phase distribution; (c) Unwrapped phase distribution as a surface mesh plot.

Figure 3. Phase profiles of the specimen displacement at $t=1000$, obtained along a horizontal line crossing the point of maximum deformation, using the 4-frame phase shifting algorithm and re-referencing every 5 frames $\left(\tau_{\mathrm{r}}=5\right)$ : (a) With vibration isolation and the vacuum pump switched off; (b) With no vibration isolation and the vacuum pump running.

Figure 4. Phase profiles obtained after 250, 500, 750 and 1000 frames with the 4-frame phase shifting algorithm and $\tau_{\mathrm{r}}=5$ with no vibration isolation and the vacuum pump running.

Figure 5. Phase profiles obtained for $t=1000$ with the 4 -frame phase shifting algorithm for $\tau_{\mathrm{r}}=0,30,60,120,240$ and $\infty$ (inf) with no vibration isolation and the vacuum pump running.

Figure 6. Phase profiles obtained for different phase shifting algorithms and $\tau_{\mathrm{r}}=60$ with no vibration isolation and the vacuum pump running. 


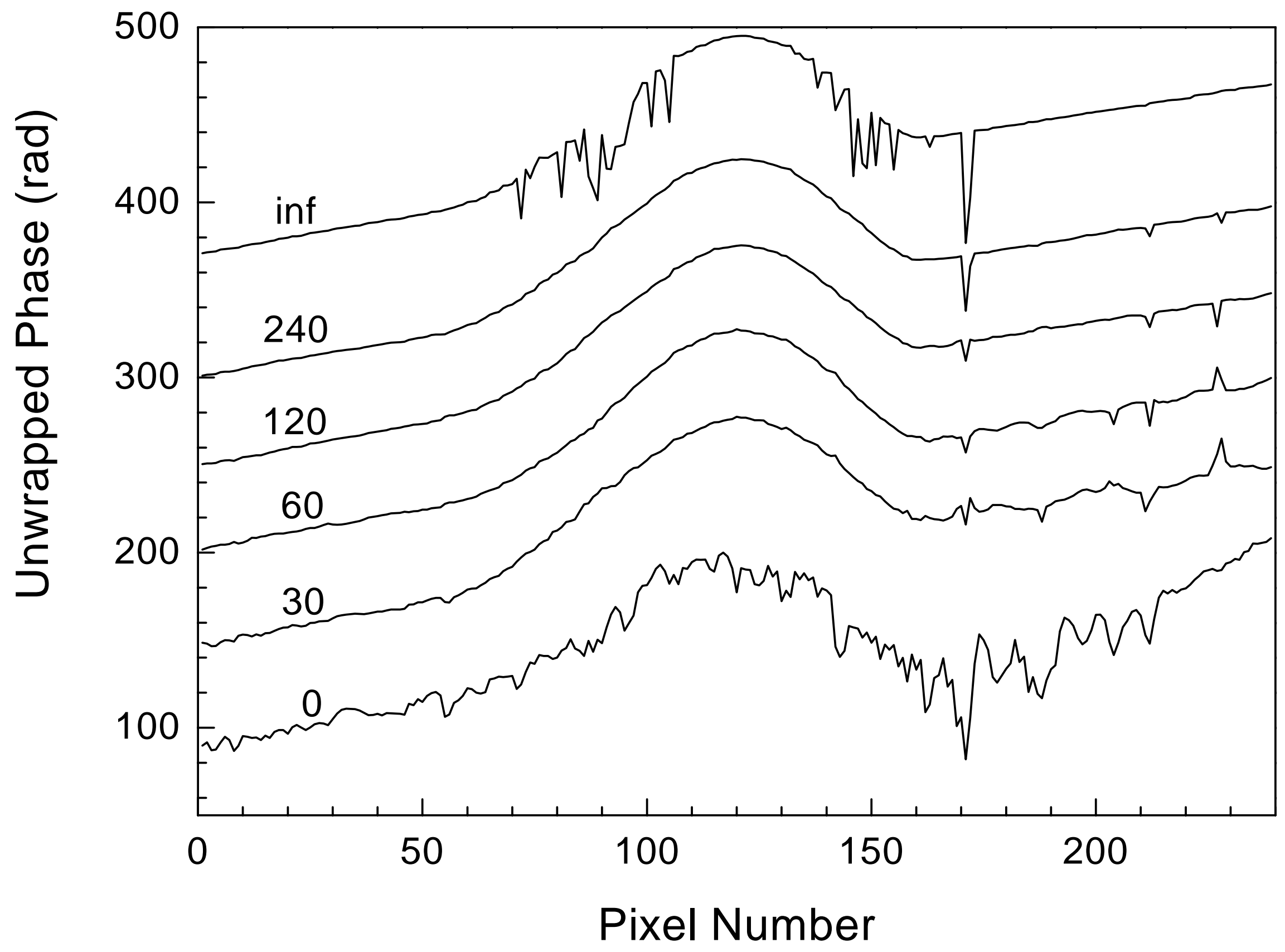




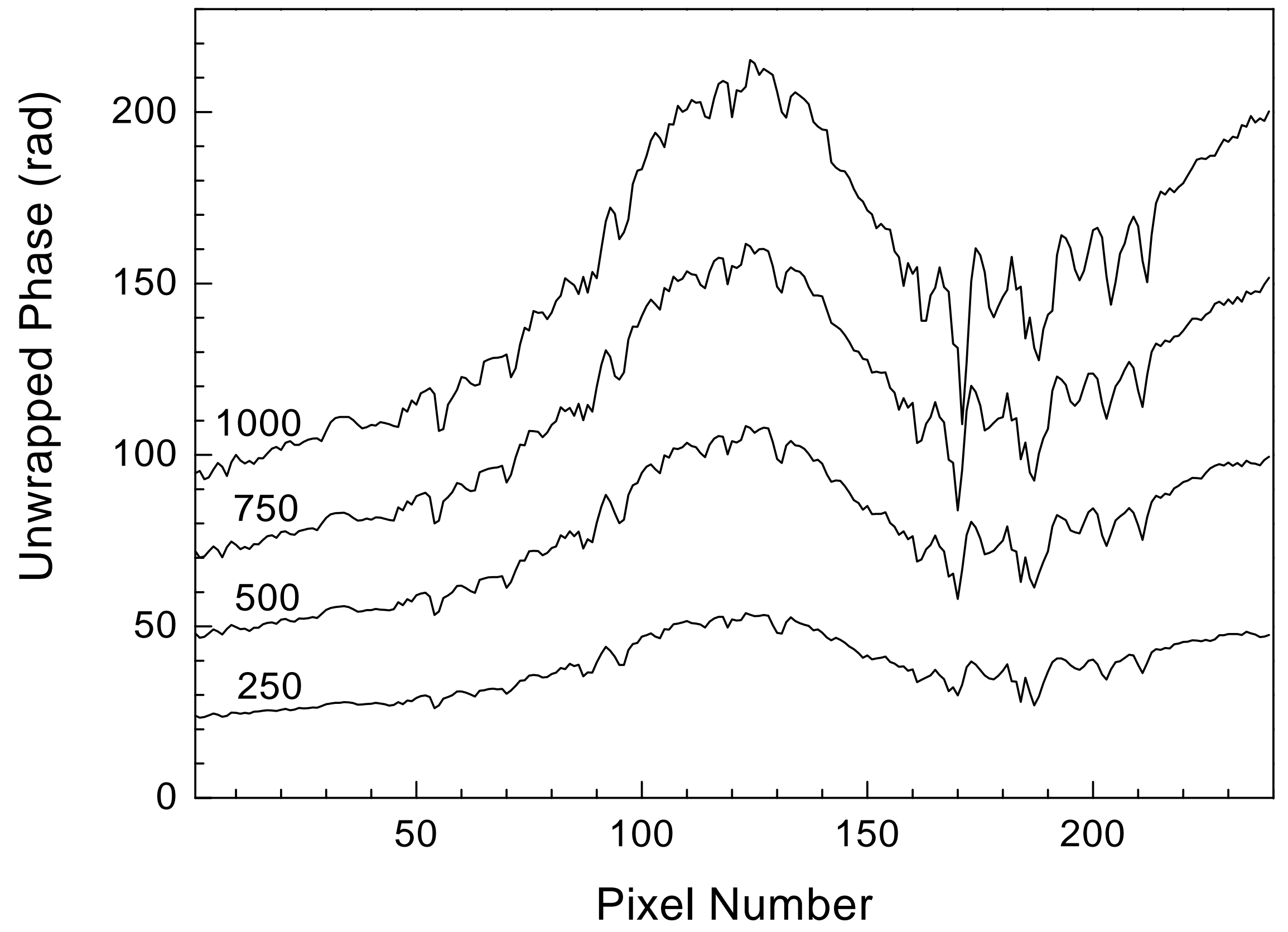




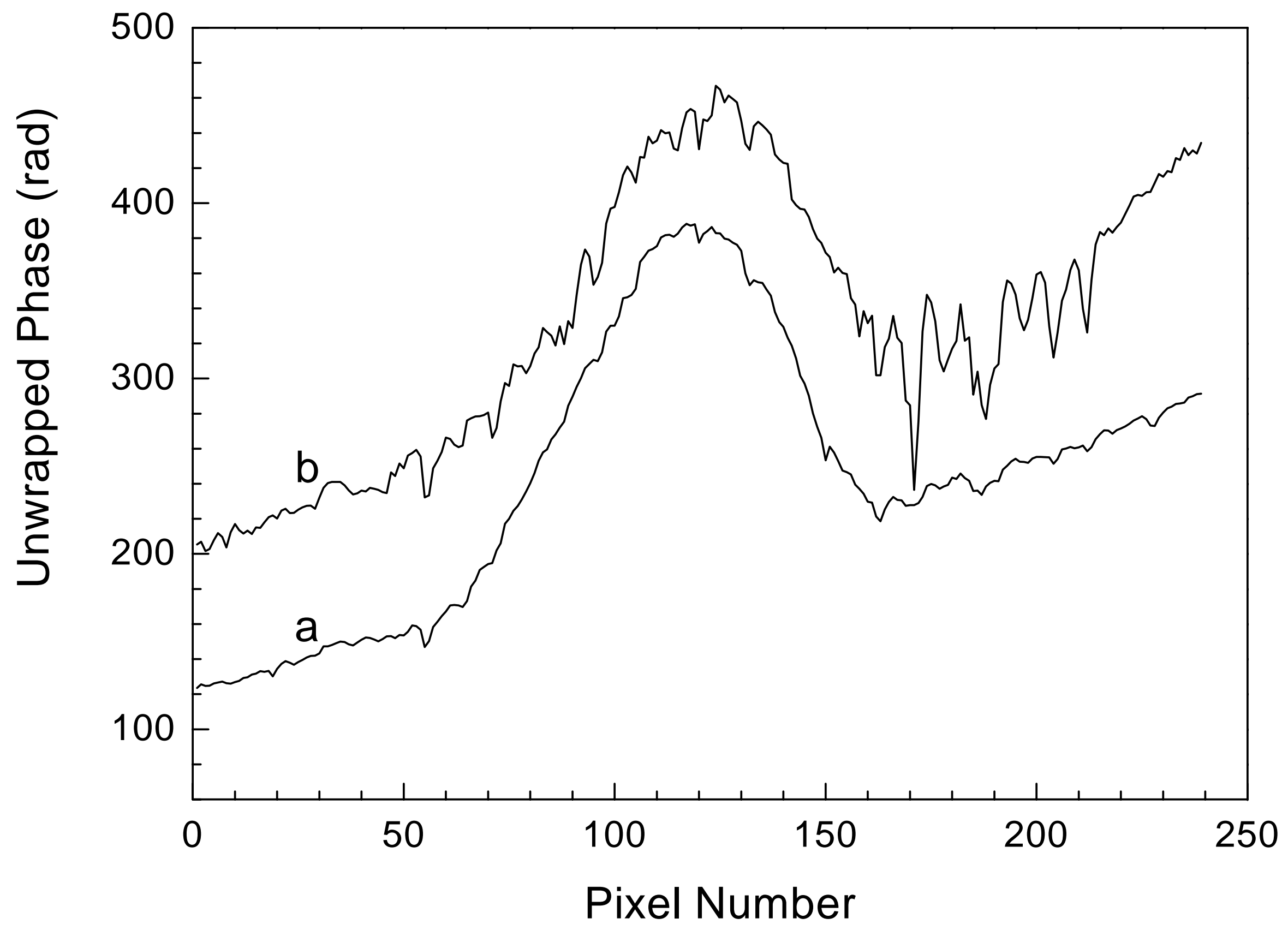




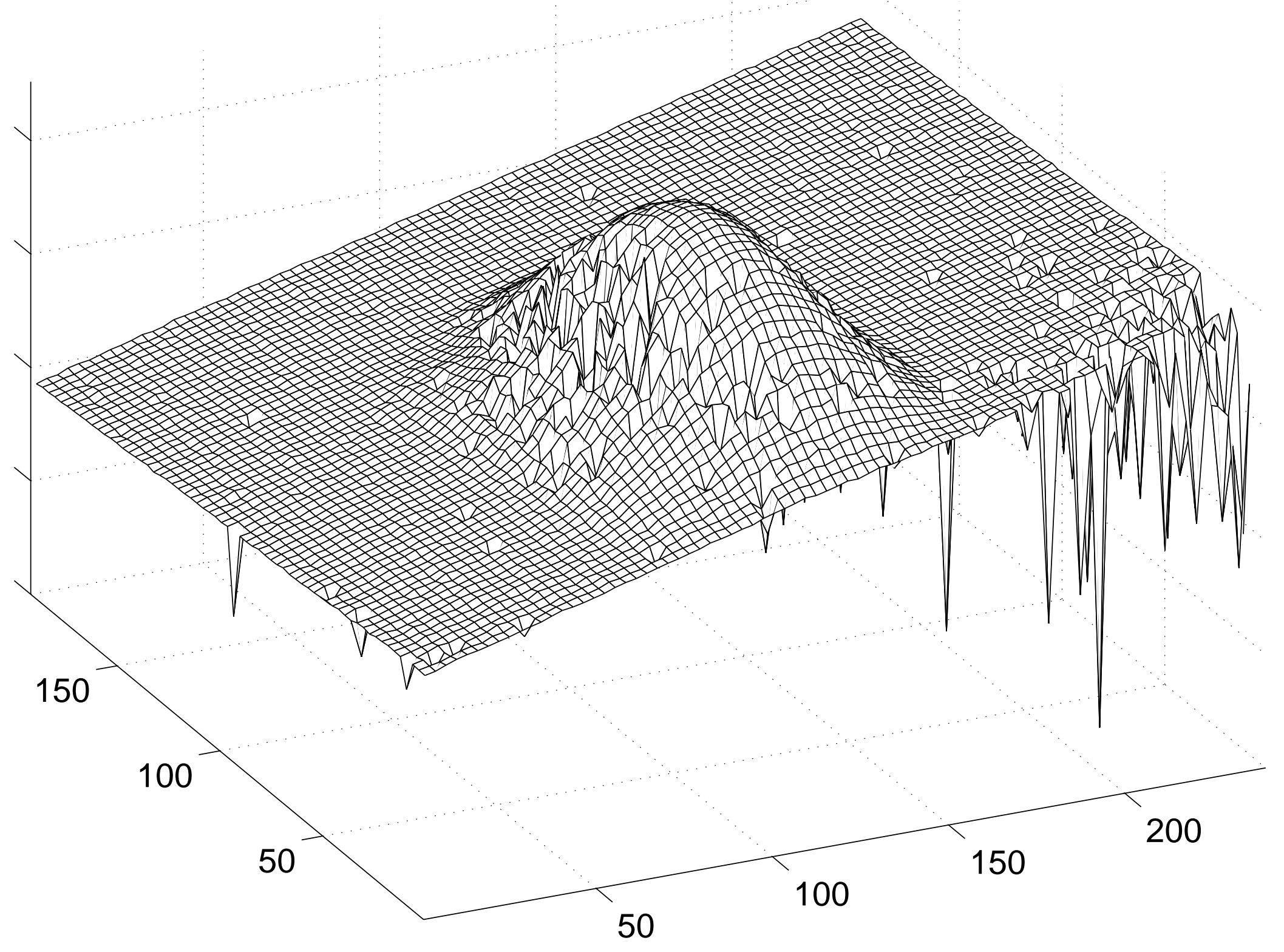




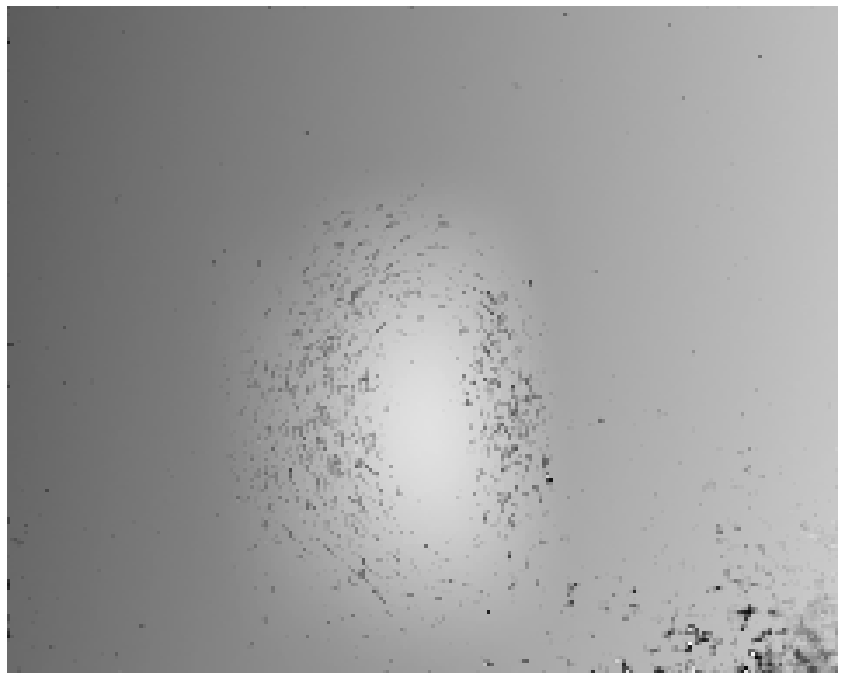




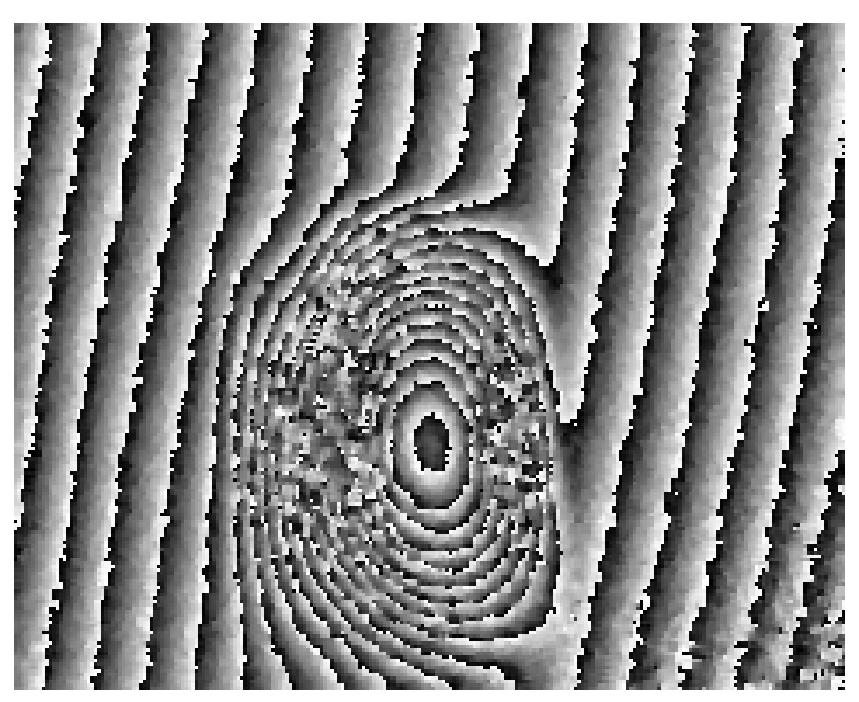




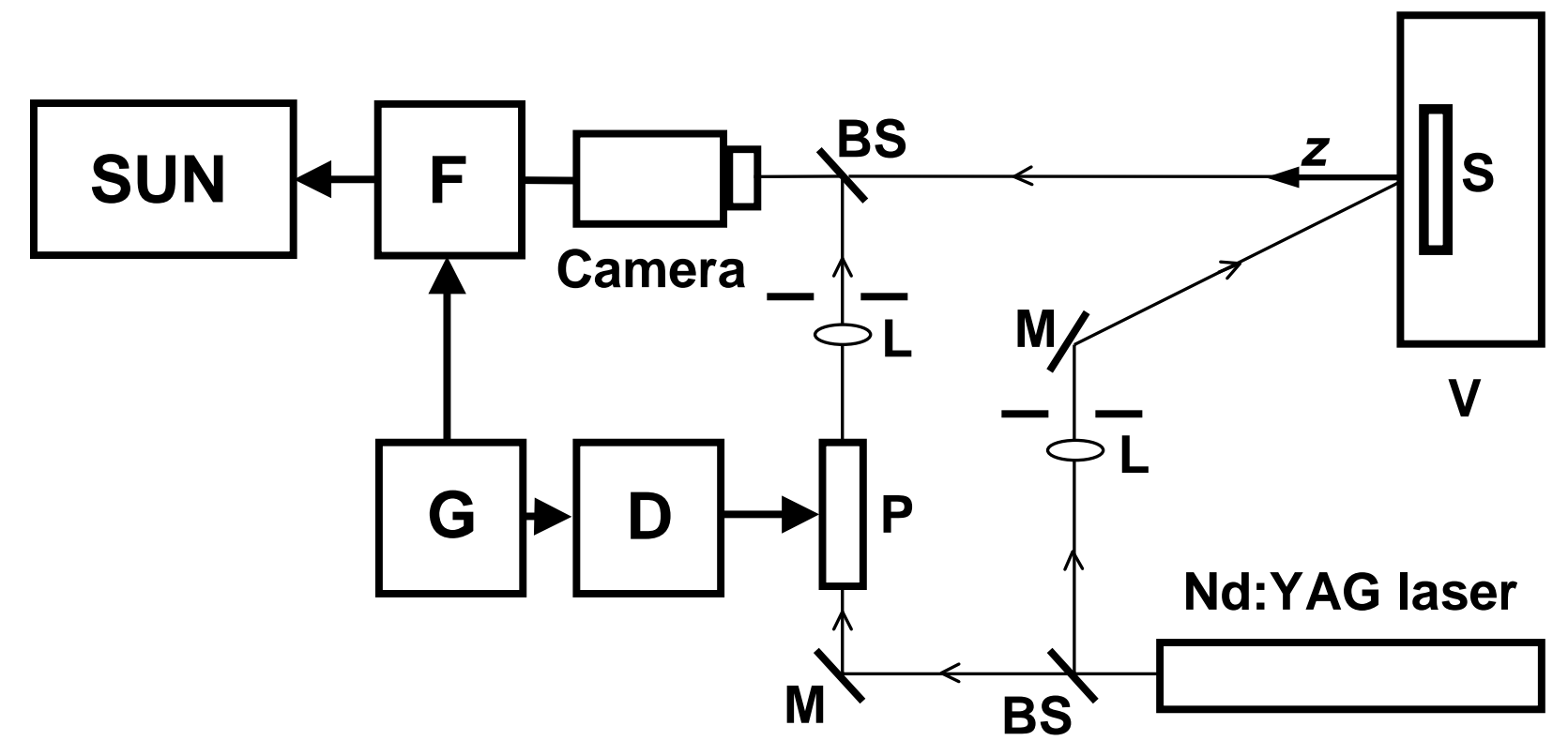




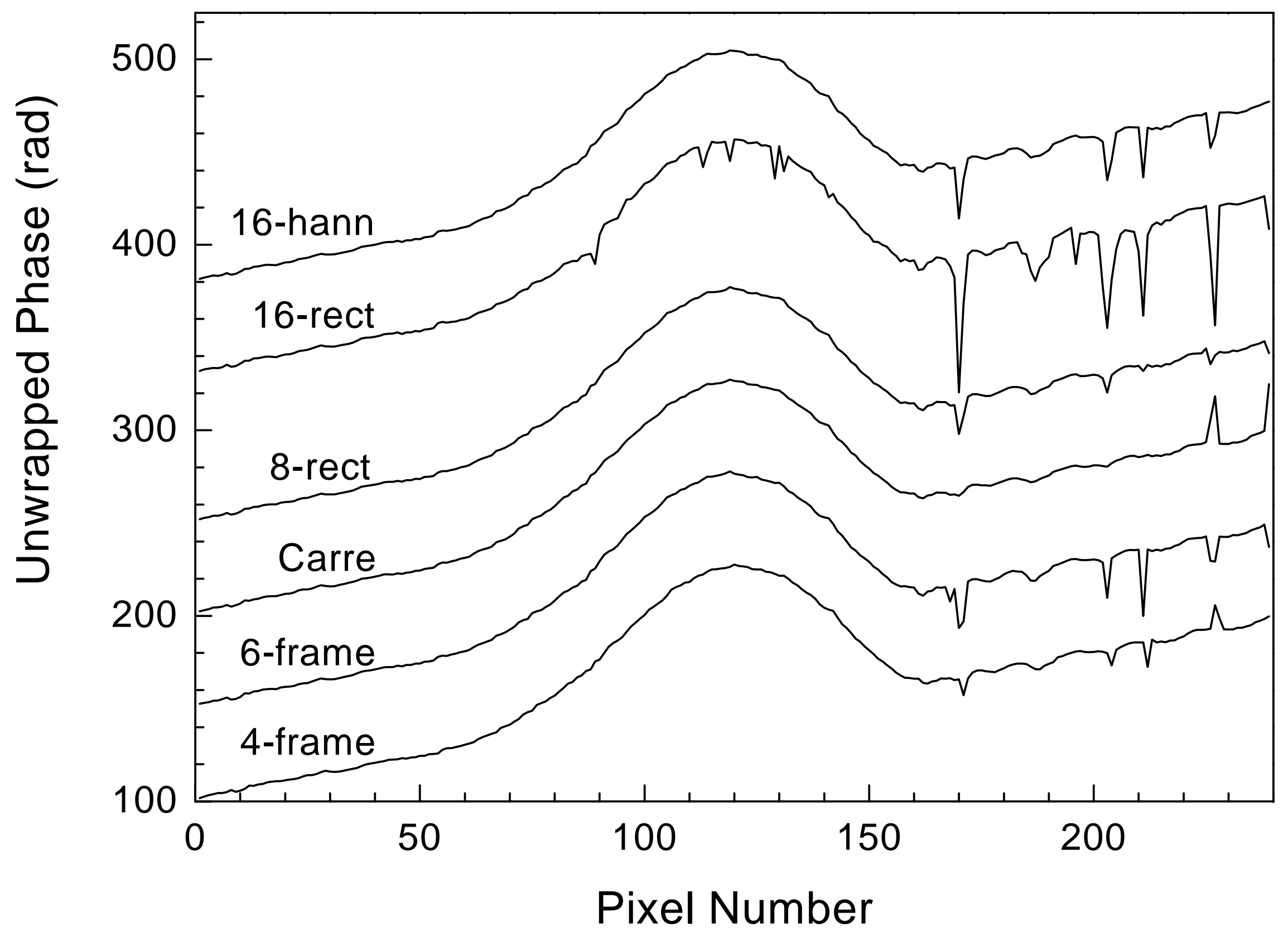

